\title{
Biologic characterization of a secondary glioblastoma with extracranial progression and systemic metastasis
}

\author{
Shigeo Ueda, ${ }^{1}$ Toshihiro Mineta, Kenji Suzuyama, Makoto Furuta, Tetsuya Shiraishi, \\ and Kazuo Tabuchi.
}

Department of Neurosurgery, Saga Medical School, Saga 849-8501, Japan

Glioblastomas rarely metastasize outside the CNS. We biologically characterized a case of secondary glioblastoma associated with extracranial progression and distant metastasis. A 42-year-old male patient was subjected to craniotomy for a left temporal tumor (astrocytoma grade II) and subsequently underwent another 3 craniotomies due to tumor recurrences. At the third craniotomy, extracranial progression was noted, and the tumor was classified as a glioblastoma. In order to pinpoint the genes expressed differentially in the intracranial primary tumor and the metastatic tumors, we used cDNA microarray. The patterns of gene expression in these 2 samples were highly similar, suggesting that the mechanism of metastasis was direct infiltration of tumor cells into extracranial blood vessels. Insulin-like growth factor binding protein-2 was overexpressed in both primary and metastatic tumors. Immunohistochemical studies of DNA-dependent protein kinase, which participates in the repair of DNA, was strongly positive in the samples obtained at the first and second operations, but the positive rates were markedly reduced in the specimens obtained at the third and fourth operations. These results suggest that insulinlike growth factor binding protein-2 and deficiency of DNA-dependent protein kinase proteins promoted tumor progression in the present case. Neuro-Oncology 5,

\section{Received 24 June 2002, accepted 26 July 2001}

${ }^{1}$ Address correspondence and reprint requests to Shigeo Ueda, M.D., Department of Neurosurgery, Saga Medical School, 5-1-1 Nabeshima, Saga 849-8501, Japan.

${ }^{2}$ Abbreviations used are as follows: DNA-PK, DNA-dependent protein kinase; DNA-PKcs, DNA-PK catalytic subunit; GBM, glioblastoma multiforme; IGFBP2, insulin-like growth factor binding protein-2.
14-18, 2003 (Posted to Neuro-Oncology [serial online], Doc. 02-016, December 4, 2002. URL http://neurooncology.mc.duke.edu)

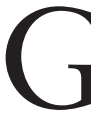
$\mathrm{BM}^{2}$ is a highly malignant tumor that accounts for about $20 \%$ of all primary brain tumors. The prognosis of GBM patients is very poor, with an average survival time of approximately 1 year (Black et al., 1991). However, extracranial metastasis is rather rare, with a frequency of only $0.44 \%$ (Smith et al., 1969). We report on a patient with secondary GBM associated with extracranial progression and distant metastasis. Subsequent to autopsy, immunohistochemical examination and an analysis of gene expression using cDNA microarray were performed to investigate possible mechanisms of tumor progression and extraneural spread of this tumor.

\section{Case Study}

\section{History}

In September 1991, a 42-year-old man developed headache, vomiting, and generalized convulsions. A CT scan showed a nonenhancing tumor in the left temporal lobe. Craniotomy was performed with gross total resection of the grade II astrocytoma. Two years later, regrowth of the tumor was noted and another craniotomy with tumor resection was performed. Again, the pathologic diagnosis was grade II astrocytoma. Postoperatively, the patient was treated with 5,000 cGy of external beam radiation, and the postoperative course was satisfactory. However, in October 1997, an s.c. tumor was discovered in the left retroauricular region. MRI revealed tumor regrowth with 


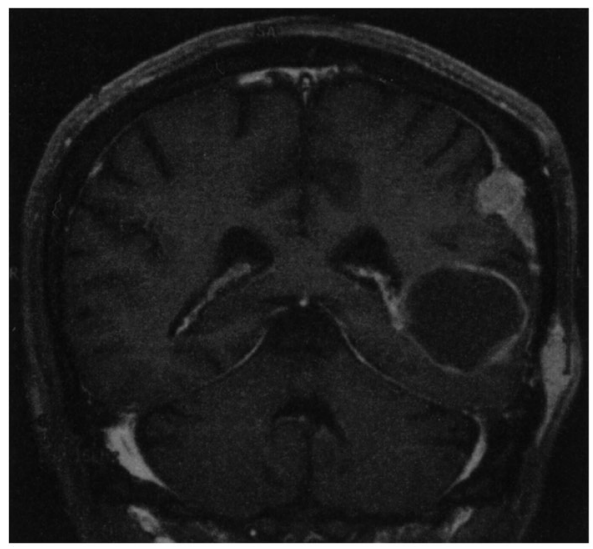

$\mathbf{A}$

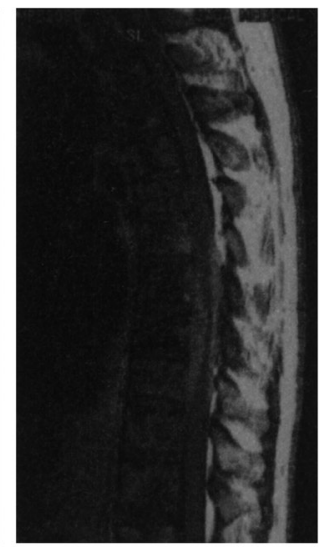

B

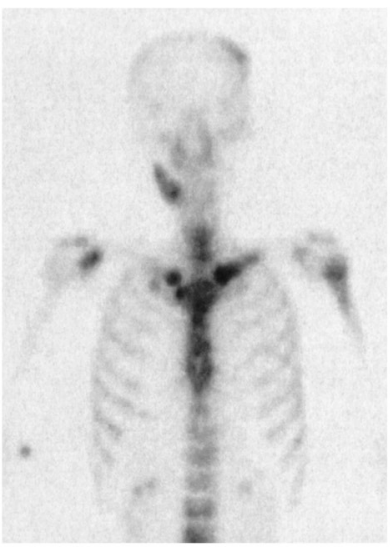

C

Fig. 1. Radiologic scans showing extracranial progression and distant metastasis in a 42-year-old man with glioblastoma. A. Gadoliniumenhanced $\mathrm{T}_{1}$-weighted MRI of the brain revealing a ring-shaped enhanced mass in the left temporal lobe, a nodular thickening of the dura, and an enhanced tumor around the left auricle (coronal view). B. Gadolinium-enhanced $\mathrm{T}_{1}$-weighted MRI of the thoracic spine showing enhanced extradural masses compressing the spinal cord (sagittal view). C. Bone scan revealing multifocal bone metastasis.

a ring-shaped enhancement, together with a nodular thickening of the dura. In addition, there was a gadolinium-enhancing tumor around the left auricle (Fig. 1A). We diagnosed the patient with a regional recurrence of the tumor associated with extracranial progression. We performed a third craniotomy followed by tumor resection, and the postoperative pathologic diagnosis was GBM. Postoperatively, the patient received 3 cycles of chemotherapy with ranimustine (MCNU). However, the left temporal s.c. and i.c. tumors grew in size. In September 1998 , a fourth craniotomy with a transplantation of free skin flap was performed with resection of i.c. and s.c. tumors. However, growth of the tumor progressed, and we discovered metastasis of the tumor to the cervical lymph nodes and thoracic vertebrae. Spinal MRI revealed an extradural tumor enclosing the spinal cord at the level of T6-10 (Fig. 1B). In addition, bone scintigraphy demonstrated an intense uptake of radiotracer in pathologic areas involving the left clavicle, left first rib, and thoracic vertebrae, thus indicating multifocal bone metastases (Fig. 1C). The patient's respiratory condition deteriorated, and he died 7 years and 7 months after the first surgery. Postmortem examination revealed glioblastoma involving the left temporoparietal lobes. The metastases were located in the following areas: cervical spinal cord, both lungs, epicardium, right kidney, pancreas, liver, left cervical and auricle soft tissue, bones (left clavicle, left ribs, cervical and thoracic vertebrae), and multiple lymph nodes.

\section{cDNA Microarray}

To pinpoint the genes expressed differentially in the i.c. primary tumor and in the metastatic loci, we used cDNA microarray to examine samples obtained from the primary tumor at the fourth surgery and from a metastatic tumor in a cervical lymph node obtained at early autopsy
(2.0 h postmortem). For this examination, the commercially available IntelliGene Human Apotosis CHIP Version 1.1 (Takara, Tokyo, Japan) was used, and the expression levels of 164 genes associated with apoptosis and cell-cycle regulation were analyzed. Calculations for preparing a spot template and for evaluating the background level were performed using Quant Array (GSI Lumonics, Watertown, Mass.). In both primary and metastatic tumor samples, IGFBP2 and BAD (BCL2 antagonist of cell death) were expressed at more than twice the level of internal controls (Table 1). Differential gene expression of i.c. and metastatic tumor tissues was recognized with regard to several genes, but in most genes the difference was not significant.

\section{Immunohistochemistry}

After each operation and autopsy, the excised tumor samples underwent histopathology and immunoreactive stain-

Table 1. Genes shown to be up-regulated by CDNA microarray analysis in intracranial and extracranial glioblastoma compared with control (b-actin)

\begin{tabular}{cc} 
CDNA expression array: Genes & Up-regulated expression \\
\hline Intracranial GBM & Intracranial vs control \\
IGFBP2 & 2.228 \\
$B A D$ & 2.184 \\
Extracranial GBM & Extracranial vs control \\
$M C L 1$ & 3.251 \\
NME2 & 2.820 \\
$B A D$ & 2.486 \\
IGFBP2 & 2.108 \\
\hline
\end{tabular}

Abbreviations: $B A D, B C L 2$ antagonist of cell death; $M C L 1$, myeloid cell leukemia sequence1: NME2, nonmetastatic cells-2. 

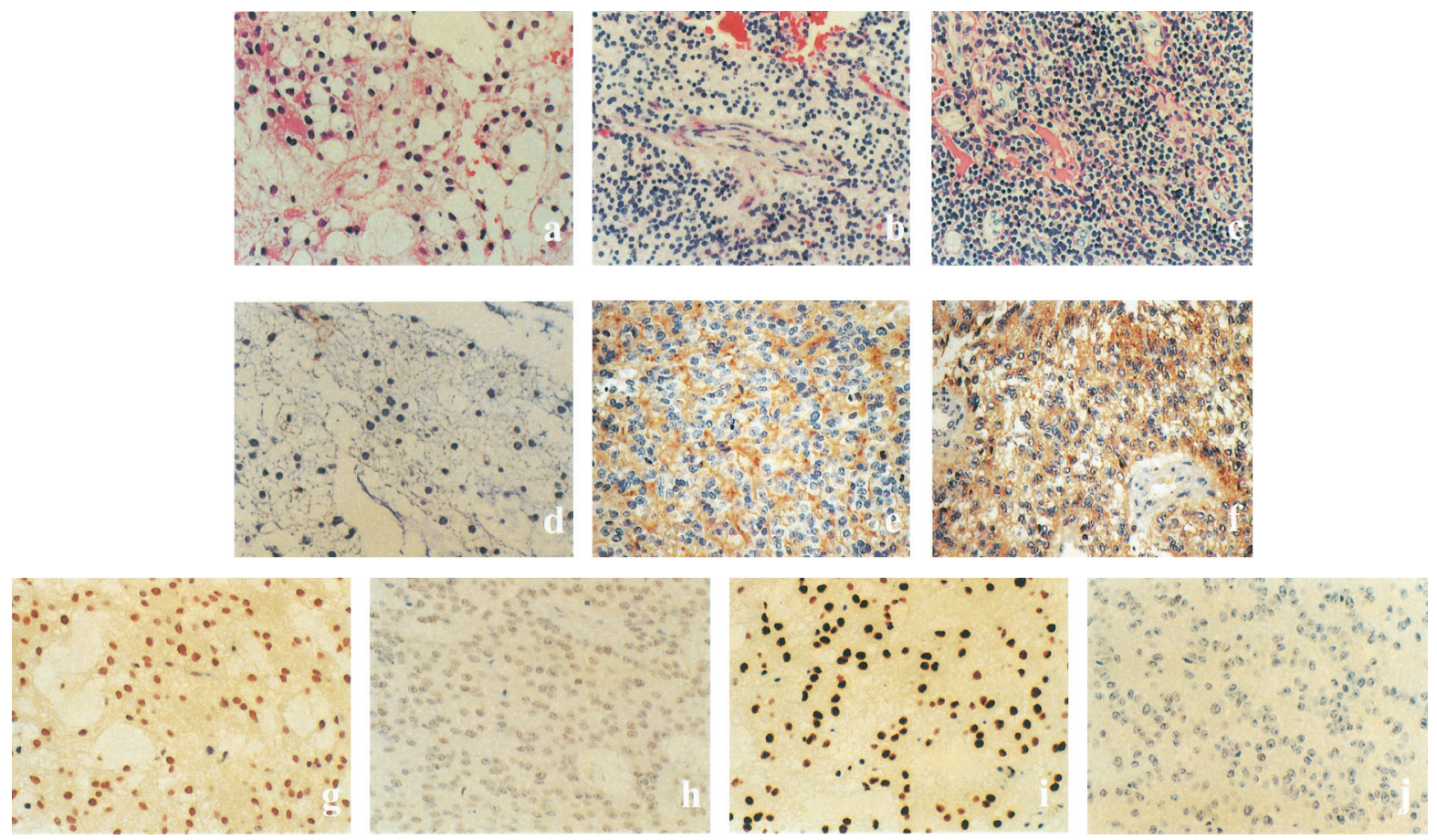

Fig. 2. Histopathology and immunoreactive staining of excised tumor samples obtained at operations and autopsy. Upper: Hematoxyline and eosin stainings of the tumor samples at the first operation (A), the fourth operation (B), and autopsy (C). Middle: Immunoreactive stainings for IGCBP2 of the tumor samples at the first operation (D), the fourth operation (E), and autopsy (F). IGFBP2 immunoreactivity was positive in samples obtained at the fourth operation and autopsy. Lower: Immunoreactive stainings for Ku86 and DNA-PKcs of the tumor samples at the second ( $\mathrm{G}$ and $\mathrm{I}$ ) and the third $(\mathrm{H}$ and $\mathrm{J}$ ) operations. $\mathrm{G}$ and $\mathrm{H}$ were immunostained for Ku86, whereas I and J were stained for DNAPKcs. In tumor samples obtained at the third operation, the positive rates for Ku86 and DNA-PKcs were reduced.

ing (Fig. 2). Immunoreactivity for glial fibrillary acidic protein was positive in all operative samples. Vimentin immunoreactivity was positive only in the samples obtained at the third and fourth operations. The MIB-1 positive rate was $4 \%$ at the first surgery, $15 \%$ at the second surgery, $22 \%$ at the third surgery, and $28 \%$ at the fourth surgery, showing that the positive rate progressively increased. Immunoreactivities of $\mathrm{p} 53, \mathrm{mdm} 2$, and p16 were all negative in all samples. IGFBP2 immunoreactivity was positive in all operative and autopsy samples except for samples obtained at the first and second operations (Fig. 2D-F). Furthermore, we examined immunoreactivity of DNA-PK. DNA-PK consists of a catalytic subunit, DNA-PKcs, combined with Ku70 and Ku86 proteins and participates in the repair of DNA double-strand breaks. Immunoreactivities of DNA-PKcs, Ku70, and Ku86 were strongly positive in the samples obtained at the first and second surgeries, but the positive rate was reduced in samples obtained at the third surgery (Fig. 2G-J). The results of the immunohistochemical examinations are summarized in Table 2.

\section{Discussion}

Generally, extracranial metastases of GBMs occur after craniotomy or trepanation procedures, and such metastases are attributed to infiltration of tumor cells into sur- gical defects and extracranial blood vessels (Liwnicz et al., 1979). In some cases, tumor cells progress extracranially through mechanical escape routes such as ventriculoatrial shunts (Wakamatsu et al., 1972). In the present case, s.c. tumor infiltration was recognized at the time of the third operation. It is highly probable that the above-mentioned mechanisms contributed to the state of generalized metastasis. On the other hand, distant metastases have also been observed in cases where no craniotomies had been performed (Vural et al., 1996; Yanagawa et al., 1996). Recently, Park and his colleagues evaluated genetic alterations from both primary and metastatic samples of 6 patients with GBM (Park et al., 2000). They confirmed the emergence of neoplastic subclones in metastatic foci, suggesting that some of the metastases arose from genetically altered subclones that were presumably only minor populations in the primary tumor or were not represented in the primary tumor.

In the present study, we used the cDNA microarray technique to analyze gene expression in i.c. and metastatic tumor samples. We found several genes expressed differentially in these 2 samples. Most differences, however, were not significant. In total, our results suggest that the mechanism of distant metastasis in the present case was direct extracranial infiltration of the tumor.

To pinpoint gene expression as related to tumor progression, we performed immunostaining of excised tissue and autopsy samples. The P53 gene is widely known as a 
Table 2. The status of glial fibrillary acidic protein, vimentin, Ku70, Ku86, DNA-PKcs, and IGFBP2 immunoreactivities and MIB-1 score

\begin{tabular}{lccccccc} 
Sample & GFAP & Vimentin & MIB-1 $(\%)$ & KU70 & KU86 & DNA-PKCS & IGFBP2 \\
\hline 1st operation & +++ & - & 4 & +++ & +++ & +++ & - \\
2nd operation & +++ & - & 6 & +++ & +++ & +++ & - \\
3rd operation & +++ & +++ & 22 & +++ & ++ & + & +++ \\
4th operation & +++ & +++ & 28 & ++ & - & - & +++ \\
\hline
\end{tabular}

Abbreviation: GFAP, glial fibrillary acid protein.

The percentage of neoplastic cells with nuclear or cytoplasmic immunoreactivity for GFAP, vimentin, Ku70, Ku86, DNA-PKcs, and IGFBP2 were recorded as - when only occasional ( $<5 \%$ ) tumor cells were positive, + when $5 \%-25 \%$ were positive, ++ when $25 \%-50 \%$ were positive, and +++ when $>50 \%$ were positive. MIB- 1 scoring was accomplished by determining the percentage of positive nuclei after counting 1,000 tumor cells.

gene related to the genesis of secondary GBM (del Arco et al., 1993; Sure et al., 1997; Watanabe et al., 1996). Although it has been reported that mutations of P53 occur in more than $65 \%$ of secondary GBM samples (Kleihues et al., 1999; Nakamura et al., 2001a, b), such mutations were absent in all samples of the present case obtained at the first to fourth operations. Similarly, mutations of MDM2 and P16 were absent in all samples. Possible P16 promoter methylation, however, was not determined (Nakamura et al., 2001a, b).

Recently, several investigators identified overexpression of IGFBP2 in glioblastoma by using the cDNA microarray technique (Fuller et al., 1999; Sallinen et al., 2000). In the present set of experiments, in which the cDNA microarray technique and immunohistochemistry were used, we confirmed the overexpression of IGFBP2 in intracranial and extracranial GBM specimens. The present results suggest that IGFBP2 contributes to the tumor progression of glioblastoma.

Furthermore, the breakdown of DNA repair mechanisms has been suggested as one of the causes of malignant transformation. DNA repair mechanisms are subdivided into 2 categories: nonhomologous end-joining and homologous recombination. A hallmark of nonhomologous end-joining is that, in contrast to homologous recombination, sequence homology between the 2 recombining DNA double strands is not required. Although the exact mechanism and the regulation of nonhomologous end-joining remain to be elucidated, DNA-PK plays a crucial role. DNA-PK is composed of a kinase element known as DNA-PKcs and a DNA binding element known as $\mathrm{Ku}$, which is itself a heterodimer composed of Ku70 and Ku86. The Ku70 and Ku86 heterodimer binds to free DNA ends at the DNA double-strand break and recruits DNA-PKcs, activating its kinase activity. A complex of proteins, including DNA ligase IV, is then recruited and interacts with DNA-PK to bring about the repair of the DNA doublestrand break. Recently, decreased expression of DNA-PK has been reported to cause tumorigenesis (Kurimasa et al., 1999; Rigas et al., 2001). In the present case, all of the nonhomologous end-joining-related proteins were strongly positive in the samples obtained at the first and second operations, but the positive rates were reduced at the third and fourth operations. Collectively, the results suggest that a functional deficiency of DNA-PK occurs during the tumor progression of this case and may play a role in malignant transformation of glioma.

In conclusion, a case of secondary glioblastoma associated with extracranial progression and distant metastasis has been reported. Experiments using cDNA microarray to test for genes that were expressed differentially in i.c. and metastatic tumor samples showed highly similar results, suggesting that the mechanism of metastasis was direct infiltration of the tumor cells into extracranial blood vessels.

\section{References}

Black, K. L., Mazziotta, J. C., and Becker, D. P. (1991) Brain tumors. West. J. Med. 154, 186-197.

Del Arco, A., Garcia, J., Arribas, C., Barrio, R., Blazquez, M. G., Izquierdo, J. M., and Izquierdo, M. (1993) Timing of p53 mutations during astrocytoma tumorigenesis. Hum. Mol. Genet. 2, 1687-1690.

Fuller, G. N., Rhee, C. H., Hess, K. R., Caskey, L. S., Wang, R., Bruner, J. M., Yung, W. K., and Zhang, W. (1999) Reactivation of insulin-like growth factor binding protein 2 expression in glioblastoma multiforme: A revelation by parallel gene expression profiling. Cancer Res. 59, 4228-4232.

Kleihues, P., and Ohgaki, H. (1999) Primary and secondary glioblastoma: From concept to clinical diagnosis. Neuro-Oncology [serial online], Doc. 98-18, January 19, 1999. URL neuro-oncology.mc.duke.edu. Neurooncol. 1, 44-51.

Kurimasa, A., Ouyang, H., Dong, L. J., Wang, S., Li, X., Cordon-Cardo, C., Chen, D. J., and Li, G. C. (1999) Catalytic subunit of DNA-dependent protein kinase: Impact on lymphocyte development and tumorigenesis. Proc. Natl. Acad. Sci. U. S. A. 96, 1403-1408.

Liwnicz, B. H., and Rubinstein, L. J. (1979) The pathways of extraneural spread in metastasizing gliomas: A report of three cases and critical review of the literature. Hum. Pathol. 10, 453-467.

Nakamura, M., Watanabe, T., Klangby, U., Asker, C., Wiman, K., Yonekawa, Y., Kleihues, P., and Ohgaki, H. (2001a) p14ARF deletion and methylation in genetic pathways to glioblastomas. Brain Pathol. 11, 159-168.

Nakamura, M., Yonekawa, Y., Kleihues, P., and Ohgaki, H. (2001b) Promoter hypermethylation of the RB1 gene in glioblastomas. Lab Invest. 81, 77-82.

Park, C. C., Hartmann, C., Folkerth, R., Loeffler, J. S., Wen, P. Y., Fine, H. A., Black, P. M., Shafman, T., and Louis, D. N. (2000) Systemic metastasis in glioblastoma may represent the emergence of neoplastic subclones. J. Neuropathol. Exp. Neurol. 59, 1044-1050.

Neuro-Oncology $\square$ JANUARY 2003 
Rigas, B., Borgo, S., Elhosseiny, A., Balatsos, V., Manika, Z., Shinya, H., Kurihara, N., Go, M., and Lipkin, M. (2001) Decreased expression of DNA-dependent protein kinase, a DNA repair protein, during human colon carcinogenesis. Cancer Res. 61, 8381-8384.

Sallinen, S. L., Sallinen, P. K., Haapasalo, H. K., Helin, H. J., Helen, P. T., Schraml, P., Kallioniemi, O. P., and Kononen, J. (2000) Identification of differentially expressed genes in human gliomas by DNA microarray and tissue chip techniques. Cancer Res. 60, 6617-6622.

Smith, D. R., Hardman, J. M., and Earle, K. M. (1969) Metastasizing neuroectodermal tumors of the central nervous system. J. Neurosurg. 31, 50-58.

Sure, U., Ruedi, D., Tachibana, O., Yonekawa, Y., Ohgaki, H., Kleihues, P., and Hegi, M. E. (1997) Determination of p53 mutations, EGFR overexpression, and loss of p16 expression in pediatric glioblastomas. J. Neuropathol. Exp. Neurol. 56, 782-789.

Vural, G., Hagmar, B., and Walaas, L. (1996) Extracranial metastasis of glioblastoma multiforme diagnosed by fine-needle aspiration: A report of two cases and a review of the literature. Diagn. Cytopathol. 15, 60-65.

Wakamatsu, T., Matsuo, T., Kawano, S., Teramoto, S., and Matsumura, H. (1972) Extraneural metastasis of intacranial tumor. Acta Pathol. Jpn. 22, 155-169.

Watanabe, K., Tachibana, O., Sata, K., Yonekawa, Y., Kleihues, P., and Ohgaki, H. (1996) Overexpression of the EGF receptor and p53 mutations are mutually exclusive in the evolution of primary and secondary glioblastomas. Brain Pathol. 6, 217-223.

Yanagawa, Y., Miyazawa, T., Ishihara, S., Takiguchi, H., Shima, K., Terahata, S., Tamai, S., and Chigasaki, H. (1996) Pontine glioma with osteoblastic skeletal metastases in a child. Surg. Neurol. 46, 481-484.

Zhang, W., Wang, H., Song, S. W., and Fuller, G. N. (2002) Insulin-like growth factor binding protein 2: Gene expression microarrays and the hypothesis-generation paradigm. Brain Pathol. 12, 87-94. 